\title{
EL PROBLEMA DE LA SALUD EN LOS ESTADOS UNIDOS
}

\author{
Por \\ Elsie L. Montiel Ziegler*
}

\section{RESUMEN}

El alto costo de la atención a la salud es uno de los principales problemas internos que ha enfrentado la sociedad norteamericana en los últimos meses. Existe un acuerdo general acerca de la necesidad de reducir los costos sin afectar el derecho de todos los norteamericanos a la atención de sus necesidades de salud.

La crisis que afecta al sistema de salud de los Estados Unidos involucra varios aspectos: los costos han ido creciendo rápidamente, de manera que la nación gasta más del $12 \%$ de su producto interno bruto en salud (más que cualquier otra nación industrializada); hay un número cada vez más grande de ciudadanos que carecen de seguro de salud; casi todos los estados de la unión americana se quejan de déficits presupuestales causados por su participación en los programas federales MEDICAD y MBDICARB, que atienden las necesidades de atención médica de los pobres y los ancianos. Ante los urgentes llamados a una reestructuración del sistema varias propuestas han sido elaboradas. Sin embargo, todavía no se logra un consenso acerca de cómo reestructurar el sistema.

Este artículo analiza los principales problemas que ocupan el debate sobre la salud, asi como sus principales consecuencias, menciona algunas de las distintas propuestas de solución, y por último, hace un comentario acerca de la factibilidad y de los obstáculos que enfrentaría una reforma global al sistema de salud.

\section{ABSTRACT}

The rising cost of health care has been one of the most important internal issues discussed in the United States over the past months. There is a widespread agreement that something must be done to reduce costs without affecting the right of all Americans to get all the health and long-term care they need.

A number of problems are involved in the so called "Health Care Crisis": health care costs are continuing to rise rapidly, with the nation spending more than $12 \%$ of its Gross National Product on health care (more than any other industrialized nation); there is a growing number of Americans that have no health insurance to protect them against any medical emergency; almost every state of the Union is complaining about budget deficits coming from MEDICAID and MRDICARE, the federal and state financed health care programs for the poor and the elderly.

There has been an urgent call for a sweeping change in American Health Care, with several proposed solutions already on the table, but there is still no consensus on how to restructure the system. The following article analyzes the problem in its different aspects and consequences, describes some of the most relevant proposed solutions and makes a comment on the feasibility and obstacles facing any significant change on the United States Health Care System.

* Lic. en sociología en la UABC. Maestría en medicina social en la UAM-Xochimilco. Actualmente trabaja en la revista Voices of Mexico del Centro de Investigaciones sobre Estados Unidos. UNAM. 


\section{INTRODUCCIÓN}

Durante 1990 uno de los problemas sociales que preocupó a varios sectores de la sociedad norteamericana fue la atención a la salud. Legisladores, los tres niveles de gobierno — federal, estatal y local—, prestadores privados de servicios médicos, patrones, sindicatos y pacientes, por mencionar a los más relevantes, discutieron y propusieron reformas al esquema de atención a la salud en los Estados Unidos. Estas propuestas van desde simples recortes presupuestales y control de gastos, hasta un "radical" sistema nacional de salud que garantice el acceso de todos los ciudadanos norteamericanos a los servicios médicos.

La discusión sobre este problema en los Estados Unidos se centra en cuatro aspectos básicos: 1) El notable incremento en los costos de los servicios observado en la última década. 2) El efecto que lo anterior tiene en los diferentes niveles de gobierno, en tanto que parte del gasto social está dedicado a financiar servicios de salud para una parte del sector de la población de escasos recursos que no puede sufragarlos. 3) El costo que para los patrones significa el pago de seguros de salud cuyas primas se han elevado de tal manera que sólo las grandes empresas pueden enfrentar el gasto que representa asegurar a sus empleados. 4) Las consecuencias que lo anterior tiene para la población, especialmente aquella que no está asegurada y carece de recursos suficientes para hacerlo. Consecuencias en términos de: cobertura, acceso a los servicios, calidad de la atención y daños a la salud.

Como resultado de la discusión, han surgido una infinidad de propuestas de solución. Estas varían en su alcance —estatales y nacionales- en su nivel de radicalización, y en la aceptación que presuponen por parte de los grupos involucrados y de la población en general. Ahora bien, aun cuando la insatisfacción es general y la necesidad de reformas es aceptada, en el ámbito político prevalece una actitud cautelosa. Ésta se refleja en las declaraciones de legisladores tanto demócratas como republicanos en el sentido de que toda reforma debe ser cuidadosamente considerada antes de ser adoptada a nivel nacional. ${ }^{1}$ En todo caso, existe un consenso general entre congresistas y grupos de "lobbystas" en el sentido de que el debate sobre salud será un tema básico en las elecciones presidenciales de 1992 (Washington Post, 26 de febrero de 1991: A-19).

\footnotetext{
1 Aunque ello no ha impedido que los gobiemos de los estados experimenten y apliquen distintos criterios y reformas en su jurisdicción.
} 
El presente ensayo tiene como objetivo abordar la problemática descrita. Para ello, se describe brevemente las características de la prestación de servicios de salud en los Estados Unidos y las bases ideológicas que la sustentan. En ese contexto, se desarrollan los principales problemas que ocupan el debate sobre la salud y las soluciones propuestas, incluyendo la factibilidad que se le otorga a cada una de ellas. Después, se hace un comentario sobre las consecuencias que los problemas y sus soluciones presentan para la población en términos de calidad y acceso a los servicios; particularmente para aquella parte de la población que no puede pagarlos. Por último, se comenta sobre los obstáculos y la factibilidad de una reforma a la estructura de servicios de salud en los Estados Unidos.

\section{EL SISTEMA DE ATENCIÓN A LA SALUD EN LOS ESTADOS UNIDOS $^{2}$}

La atención a los problemas de salud en los Estados Unidos se encuentra organizada fundamentalmente alrededor de la práctica privada de la medicina. Esto es, no existe en dicho país un sistema nacional de salud a través del cual se coordine y regule la prestación de esos servicios; tampoco tiene el gobierno una política nacional que establezca prioridades, objetivos y metas a seguir en el área de la atención médica.

La práctica de la medicina en los Estados Unidos es, primordialmente, un monopolio ejercido por un grupo selecto de miembros de la profesión médica, protegido por el gobierno y apoyado en grupos y organizaciones relacionados con ellos: asociaciones médicas, hospitales, compañías farmacéuticas, químicas y de equipo médico, así como de un importante grupo de companías de seguros médicos que lo apoyan y comparten poderosos intereses económicos.

Justificada ideológicamente en los principios del utilitarismo, la medicina se ejerce como una práctica liberal, con ánimo individualista, en donde la atención se convierte en una mercancía accesible mediante un intercambio mercantil. El eje de toda la operación es la ganancia y por lo tanto, la atención está determinada fundamentalmente por la capacidad de pago del paciente y no por su necesidad de recuperarse. En este sentido, la intervención gubernamental en la organización de los recursos de salud

2 Es conveniente aclarar que en este apartado el objetivo no es describir el proceso económico-político que históricamente ha determinado el desarrollo de la práctica médica en los Estados Unidos y las concepciones que lo sustentan, dado que dicha tarea rebasa los alcances de este ensayo. Ello no significa ignorar el hecho de que la naturaleza, composición y funciones del sector salud en los Estados Unidos es producto de su estructura económica, asf como de su concomitante estructura política y social. 
y su uso es considerada como factor que inhibe el libre ejercicio de la profesión, así como un paso hacia prácticas socialistas consideradas indeseables en la sociedad norteamericana.

En base a lo anterior, se comprende que el grueso de los servicios es accesible al sector de la población con mayor poder adquisitivo y no al que los necesita. El carácter de la atención es principalmente de tipo curativo y rehabilitatorio, medicalizado y se presta, la mayoría de las veces, en hospitales muy sofisticados. El costo es muy elevado pues descansa en la utilización de recursos de diagnóstico y terapéutica que constituyen lo último en avance científico y tecnológico. ${ }^{3}$

En la práctica, cl sistema está organizado básicamente alrededor de un sistema de seguros de salud, con participación gubernamental en ciertas áreas (protección de ancianos, niffos y parte de los pobres). El individuo puede estar protegido por un seguro contratado de manera privada o por un seguro contratado por la empresa en la que trabaja y que es parte de sus prestaciones. El costo de los seguros depende de la cobertura contratada y de los incrementos en los costos de la atención médica. Actualmente, debido a estos incrementos, las compañías de seguros han agregado el factor de riesgo como un elemento más en la consideración de las primas a pagar. ${ }^{4}$

El código de impuestos en los Estados Unidos da un trato preferencial a las grandes empresas que contratan seguros de salud para sus trabajadores, y los planes claborados por las aseguradoras para estas empresas son relativamente más baratos que el costo de contratación de un seguro independiente. Esto afecta a los dependientes de trabajadores, trabajadores no calificados y empleados de firmas menores que no pueden pagar por una cobertura adecuada.

Por su parte, el gobierno interviene para prestar servicios de salud a través de programas específicos dirigidos a los ancianos (MEDICARE) y a los niños y pobres (MEDICAID). Ambos programas nacieron en 1965, son financiados con recursos del gobierno federal y de los gobiernos estatales y están organizados como esquemas de reembolso por servicios proporcionados por particulares. MEDICARE es un seguro hospitalario proporcionado sólo para la población de edad avanzada que recibe una pensión de

3 Este modelo de atención ha sido criticado duramente por su énfasis en la reparación del daño, más que en la prevención del mismo. Se ha cuestionado también el hecho de que la medicina, vista como negocio, tiene algunas consecuencias prácticas negativas como por ejemplo, la multiplicación de tratamientos injustificados.

El factor de riesgo está relacionado con la posibilidad de que un trabajador, debido a las condiciones en que trabaja o a un historial clínico abultado, tenga la probabilidad de requerir el servicio con una frecuencia mayor o a un costo muy elevado. (Washington Past, noviembre 22 de 1990:A-10). 
la seguridad social (Social Security Pension); el beneficiario paga una prima deducible de su pensión y el resto es financiado por los estados y la federación. Existe dentro del programa un plan adicional voluntario, que permite cubrir los gastos de cuentas médicas. MEDICAID es un programa de asistencia médica gratuita para los pobres. En octubre de 1990, el paquete presupuestal aprobado por el congreso, expandió los beneficios del programa para los nifos y ancianos pobres (New York Times, 4 de noviembre de 1990:14), sin embargo, MEDICAID no cubre a la totalidad de los pobres, debido a que, siendo un programa financiado conjuntamente estados-federación y administrado por los primeros, deja a éstos en libertad de establecer los límites mínimos de ingresos debajo de los cuales se califica para el programa. 5

Otras instituciones que cubren las necesidades de atención médica y de salud de los pobres son: hospitales públicos con pacientes externos; clínicas y consultorios de los departamentos de salud pública, estatales y locales; y programas de atención primaria apoyados con fondos federales, centros comunitarios, centros de atención para migrantes, para la población india y para los veteranos. Operan con fondos estatales, federales, financiamiento de MEDICAID, subsidios de instituciones filantrópicas y fondos de ayuda caritativa. Sin embargo, es un hecho reconocido, que la asistencia brindada por estas instituciones es de mucho menor calidad y extensión que la recibida por los grupos sociales más solventes.

Ahora bien, el sistema así organizado abre una brecha que deja sin cobertura al grupo de población que ni es elegible para recibir la protección gubernamental, ni está protegida por un seguro de salud como derecho laboral, ni puede afrontar el gasto de adquirir un seguro individual. Se calcula que esta población suma 33 millones de personas en la actualidad (Washington Post, 20 de febrero de 1991:A-4). El hecho es significativo, ya que es mínima la probabilidad de recibir atención de médicos u hospitales privados sin estar protegidos por un seguro o depositar una fianza que garantice el pago de la misma. ${ }^{6}$ Por otro lado, calificar para

5 En los años setenta, MEDICAD cubrí en su máxima capacidad al $70 \%$ de la población considerada bajo el límite de la pobreza. A fines de los ochenta el programa no alcanz $\delta$ a cubrir ni a la mitad de ésta, debido a recortes presupuestarios y alteraciones a los criterios de elección de beneficiarios (Russell, 1989:16).

En 1986, el gobiemo aprobó una ley federal (The Emergency Medical Treatment and Active Labor Act) que obliga a los hospitales a prestar servicios de emergencia a todo aquel que lo requiera, independientemente de su capacidad de pago. Sin embargo, esta ley ha encontrado oposición entre médicos y asociaciones que afirman que es inconstitucional, porque obliga a los médicos y hospitales a otorgar atención sin recibir una compensación. En este contexto, no han sido pocos los casos en los que se ha denegado el servicio a pesar del mandato legal (New York Times, 23 de marzo de 1991:A-1). 
MEDICAID implica estar en los límites de la pobreza. La otra opción son los hospitales públicos y las ayudas caritativas.

El panorama actual se encuentra dominado por el debate acerca de la solución más adecuada para el problema de los individuos sin cobertura en un sistema que favorece incrementos constantes en los costos y parece ser incapaz de controlarlos.

\section{PROBLEMAS DEL SISTEMA DE SALUD EN LOS ESTADOS UNIDOS}

\section{El problema de los costos}

Los Estados Unidos gastan más del $12 \%$ de su producto nacional bruto en el rubro salud. Poseen el liderazgo mundial en la ciencia médica: investigación sobre tratamiento de enfermedades, recursos científicos y tecnológicos para curarlas, y médicos y hospitales especializados que cuentan con capacidad para atender todo tipo de problemas. Sin embargo, entre 33 y 37 millones de personas carecen de un seguro adecuado de salud que les permita afrontar el costo del tratamiento de la enfermedad bajo este sistema.

El énfasis en la causa del problema se ha puesto en el aspecto económico: el costo de los servicios de salud en los Estados Unidos ha ido escalando en las últimas dos décadas y lo indicado es hallar soluciones tendientes a controlar su incremento.

De acuerdo con autores como Stuart M. Butler, el sistema mismo invita a la elevación de costos. Los beneficios de salud son provistos a la mayoría de los norteamericanos por sus patrones a través de seguros de gastos médicos. Estos beneficios están libres de impuestos en el caso de grandes empresas. Esto significa un gasto reducido en las primas. Por lo mismo, es poca la atención que prestan a los costos. Para cl trabajador, a su vez, los servicios son esencialmente gratuitos, por lo que no vacila en solicitarlos. Por su parte, doctores y hospitales, a sabiendas de que los pacientes pagarán poco o nada de su bolsillo, no tienen incentivos para evitar tratamientos costosos cuyo beneficio puede ser marginal. “...El resultado neto es la tendencia de los precios del área de la salud a subir rápidamente porque ni los consumidores ni los proveedores son sensibles a los costos" (Butler, 1989). Asimismo, en un sistema donde se compite por atraer al mayor número de clientes posible: médicos, clínicas y hospitales insisten en contar con todas las facilidades y adelantos tecnológicos, ello implica aumentar el gasto en las instalaciones médicas, con su consecuente impacto en los costos de los servicios (Beverly, 1987: cap. 6). 
En cuanto a los programas subsidiados con fondos gubernamentales, los administradores de éstos tampoco parecían ser sensibles a los costos individuales, en tanto que el subsidio proviene de fondos fiscales y de primas de seguros. Por su parte, los contribuyentes no tienen forma de saber qué tanto de sus contribuciones va a parar en sufragar dichos programas (Beverly, 1987: Cap. 6).

\section{El problema de los seguros de salud privados}

En los años cincuenta, los seguros privados de salud establecían sus primas de acuerdo a una llamada "tasa comunitaria". Esto es, todos los grupos empresariales o de negocios pagaban en promedio las mismas tasas y el riesgo era distribuido sobre la más amplia población posible. Dos proveedores principales de seguros dominaban el mercado: "The Blue Cross" y "The Blue Shield".

Sin embargo, el aumento del número de compañías aseguradoras, sumado a los incrementos en los costos ocurridos en las últimas dos décadas, ha transformado el sistema de seguros privados, de manera que, actualmente, favorece los planes para grandes empresas con empleados jóvenes y cuyos empleos son considerados de bajo riesgo para su salud. De esta manera, negocios pequeños y aquéllos con empleados maduros, más susceptibles de enfermar o con empleos considerados como de alto riesgo, han sido progresivamente presionados por los aseguradores comerciales que insisten en hacerles pagar primas más altas por la cobertura.

El resultado es que muchas firmas abandonan la idea de asegurar a sus empleados ante la imposibilidad de solventar el gasto que ello implica. Se calcula que de aproximadamente 30 millones de personas que carecen de cobertura, 20 millones corresponden a trabajadores y sus dependientes con empleo en negocios con menos de 25 empleados (Washington Post, 22 de marzo de 1991:A-23 y 27 de marzo de 1991:A-21). Estos trabajadores carecen de la alternativa gubernamental, esto es, de los programas subsidiados por el gobierno (MEDICARE y MEDICAID), en tanto su ingreso familiar esté por encima de la línea de pobreza fijada por el gobierno.

Un aspecto indicativo de la importancia que empieza a cobrar este problema es que tanto la cobertura como los costos de atención a la salud -anteriormente asunto menor en las negociaciones de contratos colectivos - se han convertido en impedimentos centrales para lograr acuerdos

7 La línea de pobreza oficial está actualmente en 12,092 dlls. para una familia de cuatro. Aun cuando analistas económicos indican que no es realista y que debería estar por lo menos tres mil dólares por arriba de ésta. (New York Times, 3 de septiembre de 1990: A-1). 
contractuales. Las disputas sobre estos beneficios constituyen un factor dominante en el 78\% de los casos de huelgas, involucrando a más de 1,000 trabajadores en 1989. Esto se compara con el $18 \%$ de 1986, según datos de la Unión Internacional de Trabajadores de Servicios (SEIU)(Kirk, 1990:704).

\section{El problema de los seguros gubernamentales de salud}

Como ya se mencionó, el gobierno de los Estados Unidos financia, con fondos federales y estatales, dos programas de atención a la salud MEDICAID y MEDICARE. Estos programas están intrínsecamente ligados a las denominadas políticas sociales del estado benefactor (Welfare State). ${ }^{8}$ Ambos surgieron en 1965, en un momento en que la prosperidad economica favorecía la expansión del gasto social federal. Para 1981, la participación del gasto social, respecto del gasto federal total, llegó a ser de más de $50 \%$ (Talavera, s/f). Sin embargo, entre 1981 y 1984 hubo un crecimiento explosivo del déficit fiscal, utilizado como justificación para reducir el presupuesto para programas asistenciales. Reagan argumentó que dichos programas eran impopulares pues fomentaban la dependencia y la pobreza que supuestamente pretendían aminorar.

La parálisis federal convirtió a los estados en el nuevo marco de la política social. El gobierno federal ha trasladado crecientemente los costos de salud a los estados. De esta manera, tanto MEDICAID como MEDICARE enfrentan actualmente restricciones debido a los costos que significan tanto para la federación como para los estados. La polémica en torno a estos programas se agudiza. Por un lado, los estados afirman que la carga que representan para sus finanzas se empieza a volver insostenible. Las recientes expansiones impuestas por el gobierno federal a MEDICAID (1990) implican para los estados el gasto del $14 \%$ de sus presupuestos, lo cual es muchas veces mayor al crecimiento de sus recaudaciones. A su vez, el programa MEDICARE encuentra cada vez más difícil mantener su equilibrio financiero ante la magnitud de los

8 El estado benefactor mantiene una serie de programas dirigidos a categorías de la población: para los pobres, los ancianos, los enfermos, los desempleados, los niños y los incapacitados. Dichos programas son financiados con fondos federales y/o estatales provenientes de sus recaudaciones fiscales. Los beneficios pueden ser totalmente gratuitos para el que los recibe. Este es el caso de programas como MEDICAID, ayuda a familias con hijos dependientes (AFDC), los vales de comida (Food Stamps) por mencionar los principales. Por otra parte, hay beneficios que se reciben como resultado de contribuciones deducidas de sus salarios. Este es el caso del seguro social, que representa el programa más extensivo de todos. 
costos, por lo mismo ha sido sujeto a un número cada vez mayor de restricciones de pagos a proveedores privados. ${ }^{9}$

Por otro lado, médicos y hospitales adscritos a los programas se quejan, pues cada vez es más frecuente el número de casos en los que no reciben el reembolso total por los servicios prestados, viéndose obligados a absorber el gasto. Esto resulta de un intento por reducir costos por parte de la federación al fijar cuotas de reembolso en base a "grupos de diagnóstico relacionado" (DRG). Esto es, ordenó el pago de una cuota fija por los cuidados brindados a pacientes con padecimientos similares. La objeción de los prestadores de servicios es que no toma en cuenta posibles requerimientos individuales superiores a la cuota fijada.

Lo anterior pone en duda la calidad de la atención que dichos pacientes recibirán de los proveedores privados, a la vez que hace cada vez más difícil el acceso a los servicios debido a la renuencia a aceptar enfermos bajo estos programas. Por otra parte, este problema afecta también a pacientes particulares, pues los hospitales les aumentan las tarifas, en un intento por cubrir las pérdidas causadas por los cuidados proporcionados bajo MEDICAID Y MEDICARE.

Recientemente, los hospitales recibieron de parte de la Suprema Corte el aval legal que les permita demandar a la administración estatal para forzar el pago completo por los servicios bajo el sistema MEDICAID. Sin embargo, mientras esta decisión es una gran noticia para los hospitales, para los gobiernos estatales y federal significa un obstáculo en su intento de frenar el excesivo gasto en atención médica para los pobres. Esta situación es para muchos un indicador de que el sistema de atención a la salud para los pobres está al borde del colapso, en tanto que el gobierno no puede garantizar la ayuda a todos aquellos que la requieran (Wall Street Journal, 15 de mayo de 1990:B-1).

\section{Problemas de acceso y calidad de la atención en salud}

El problema de los altos costos tiene como consecuencia el establecimiento de limitantes de acceso a los servicios y de calidad deficiente para aquéllos que carecen de capacidad de pago. De esta manera la atención a la salud se convierte en un privilegio.

9 Un reporte elaborado por el Consejo de Asesores de Seguridad Social, señala que la Medicare podría enfrentar la ruina en 15 años si no se toman medidas para corregir el serio desequilibrio entre los costos a largo plazo y las recaudaciones. (Washington Post, 2 de abril de 1991: A-19). 
La población afectada es fundamentalmente aquélla ubicada por debajo del nivel de pobreza. Sin embargo, no es la única y aumenta el número de personas de clase media que carecen de cobertura adecuada.

\section{Dificultades de acceso a los servicios}

Como se había mencionado anteriormente, las compañías aseguradoras privadas favorecen los planes de salud para las grandes empresas. Cada vez es mayor el número de negocios medianos y pequeños que adquieren pólizas de cobertura limitada o se abstienen de adquirirlas totalmente, dejando a sus empleados desprotegidos. El problema para éstos es sui generis. Su salario no es suficiente para adquirir una póliza privada, pero sí lo es para evitar que sean elegibles para la cobertura de MEDICAID. La opción en los casos de necesidad de atención médica absurda, el individuo debe esperar a ver reducido su nivel de vida hasta el límite oficial fijado para ser considerado "pobre" para así presentar su solicitud de apoyo. Esto ocurre también en los casos de enfermedades crónico-degenerativas cuando se agotan los recursos cubiertos por sus seguros de salud privados, pues no podrán ser aceptados por MEDICAID mientras no se vean reducidos a la pobreza.

Pero el ser pobre tampoco es garantía. MEDICAID nunca ha logrado atender las necesidades de todos los pobres, aun cuando desde el principio ésa era la intención. En su máxima expresión, el programa llegó a proteger a dos terceras partes de los pobres, ello fue en 1970. Actualmente, la cobertura se ha reducido hasta menos de la mitad de éstos. Esto ha ocurrido al tiempo que la proporción de pobres ha aumentado debido a problemas económicos del país.

Esta situación se ha agravado en los últimos diez años por los intentos de controlar y reducir el gasto en salud por parte del gobierno federal, pero especialmente de parte de los gobiernos estatales, pues ellos absorben la mayor parte de la carga. Estos últimos tienen libertad para fijar requisitos y procedimientos a los solicitantes. Así, los requisitos para obtener los beneficios del programa son cada vez más excluyentes y humillantes, y los procedimientos resultan en un papeleo burocrático interminable. La alternativa son los centros comunitarios, clínicas asistenciales locales o estatales dependientes del Departamento de Salud, la ayuda caritativa o la falta total de atención médica regular (Beverly, 1987).

Ahora bien, cuando se trata de una emergencia es posible acudir a las salas de urgencias de cualquiera de los hospitales del país. Desde 1986, esta medida está respaldada por una ley que obliga a médicos y hospitales a prestar atención médica de este tipo a los pacientes pobres que acudan a 
solicitarla. Sin embargo, han sido varios los casos conocidos en los que ambos han violado la ley rehusándose a atenderlos, sin tomar en cuenta el bienestar del paciente. ${ }^{10}$

\section{Deficiencias en la calidad y extensión}

El interés excesivo puesto en el control del gasto gubernamental en programas de salud afecta directamente su calidad y extensión. Los programas limitan el número de visitas al médico y los días de estancia en hospital por año y no cubren ciertas enfermedades agudas. Médicos y hospitales reducen el nivel de atención al mínimo con el pretexto de que las cuotas límite fijadas por los gobiernos estatales y/o federal no facilitan la extensión del servicio. Acusaciones de prácticas ilegales, tratamientos innecesarios, cuidados por debajo del estándar y fraude en las cuentas por cobrar, provocaron que en 1990 el Departamento de Salud y Servicios Humanos expulsara a más de 192 médicos de sus programas MEDICAID y MEDICARE (Washington Post, 27 de noviembre de 1990:H-1).

Pero estas acusaciones no son privativas de los programas financiados por el gobierno. Las aseguradoras privadas y los empleadores han empezado a cuestionar la validez del juicio médico, contratando los servicios de compañías encargadas de valorar lo apropiado de los tratamientos propuestos por los médicos, especialmente en lo referente a operaciones y análisis costosos. Aun cuando los doctores no aprueban esta práctica, las aseguradoras han encontrado que en el $10 \%$ de los casos los tratamientos fueron innecesarios. Debido a esto, se prevé que en el futuro las aseguradoras se nieguen a pagar por este tipo de tratamientos (New York Times, 24 de febrero de 1991).

\section{Problemas de morbi-mortalidad entre la población}

Los pobres, los desempleados, las mujeres cabeza de familia, los niños y los ancianos son las víctimas principales de la problemática descrita arriba, y ello se refleja en sus condiciones de salud-enfermedad.

Los habitantes de los barrios más pobres de las ciudades viven en islas de epidemias, enfermedades y muertes prematuras. La salud de los pobres urbanos empeoró a fines de los ochenta y ha alcanzado una condición crítica. Se afirma que la culpa la tienen las condiciones de pobreza causadas por el deterioro económico y la falta de servicios médicos

${ }^{10}$ La ley referida es la "Emergency Medical Treatment and Active Labor Act", 1986, (New York Times, 23 de marzo de 1991:A-1). 
adecuados. En los últimos tres años se han incrementado los casos de sarampión, tosferina, tuberculosis, hepatitis A, sífilis, gonorrea, paperas, complicaciones e infecciones del oído, así como el SIDA. Ahora bien, aun cuando los casos no sean estadísticamente significativos, muchas de esas enfermedades eran consideradas como erradicadas y de hecho son inexistentes en barrios de clase media y alta (New York Times, 24 de diciembre de 1990:A1). El número de muertes relacionadas con el sarampión en 1990 (64) fue el más alto desde 1971. Entre 1988 y 1990, el número de casos reportados se incrementó bruscamente de menos de 5,000 a más de 24,000, situación que no se presentaba desde 1978 (New York Times, 29 de noviembre de 1990:A-1).

Los pobres no tienen atención preventiva, las vacunas no son siempre accesibles y su dieta es inadecuada. Ello se combina para incrementar sus tasas de morbi-mortalidad por enfermedades prevenibles o curables. El énfasis en las condiciones de vida como relacionadas con los problemas de salud de la población no es el enfoque predominante en los estudios sobre salud en los Estados Unidos. La mayoría de las estadísticas toman en cuenta la raza como un factor determinante y omiten registrar datos referentes al ingreso.

Sin embargo, ambos aspectos están relacionados. La proporción de negros e hispánicos en la pobreza es de 30.7 y $26.2 \%$, mientras la proporción de blancos es de $10 \%$. La tasa de mortalidad de la población negra es mayor a la de la población blanca. La esperanza de vida al nacer de los bebés negros tiene una diferencia de 6 años con respecto de los bebés blancos (69.2 años y 75.6 años). La tasa de mortalidad de los bebés negros es casi el doble respecto a la de los bebés blancos (17.2 y 8.3 por cada 1,000 nacimientos), a la vez, los primeros tienen más probabilidad de nacer con bajo peso que los segundos (Washington Post, 30 de noviembre de 1990:A-3). Los hispánicos visitan al médico con menor frecuencia que los blancos y los negros, presentan mayor número de casos de enfermedades como diabetes y SIDA, y son el grupo con menor probabilidad de contar con un seguro de salud principalmente porque son pobres (32\% de la población hispánica carece de seguro de salud respecto del $10.2 \%$ de la población blanca y $20 \%$ de la negra)(Washington Post, 15 de enero de 1991; health suplement:7).

Otro dato que apunta hacia el empeoramiento de las condiciones de salud de los pobres es el decremento en el número de visitas a las salas de emergencia de los hospitales, como es el caso de la capital del país. La razón es que para muchos pacientes pobres y carentes de seguro las salas de emergencia son la fuente primaria de atención médica básica. Pero la reticencia de los hospitales privados a aceptar a estos pacientes ha provo- 
cado que éstos se abstengan de acudir en busca de ayuda. Los oficiales de salud de la ciudad de Washington consideran lo anterior como un indicador de la inadecuada atención que está recibiendo la población de escasos recursos con un sistema de atención diferenciado para los pobres respecto al de los ricos (Washington Post, 8 de abril de 1991:D-1).

La paradoja en este caso es el hecho de que hay un exceso de infraestructura hospitalaria en los Estados Unidos. De hecho, un tercio de todas las camas de hospital se encuentran permanentemente vacías y en muchas comunidades existe una duplicación de equipo (Washington Post, 28 de febrero de 1991:A-18).

\section{PROPUESTAS DE SOLUCIÓN AL PROBLEMA DE LA SALUD}

En la búsqueda de solución a los problemas planteados han surgido diferentes propuestas, de las cuales mencionaré sólo algunas por ser las más discutidas o polémicas.

A) La propuesta más radical insiste en la necesidad de reformar el sistema en general, institucionalizando un seguro nacional de salud. Bajo este sistema todos los habitantes del país recibirían un seguro federal que cubriría: cuentas de médicos y hospitales, cuidados a largo plazo, recetas y trabajos dentales. Los pacientes serían libres de elegir médicos y hospitales, y estos últimos tendrían que aceptar que el gobierno pagara el costo total de la atención, sin cargos adicionales para el paciente. El financiamiento del programa se haría con fondos federales provenientes de los impuestos.

Esta propuesta cuenta con el apoyo de un grupo de representantes demócratas, de organizaciones ciudadanas como "Acción Ciudadana" (Citizen Action), de 10 importantes sindicatos y de grupos de médicos como el "Médicos por un Programa Nacional de Salud" (Physicians for a National Health Program). Sin embargo, grupos de interés muy poderosos, respaldados por el mismo secretario de salud de los Estados Unidos, Dr. Louis W. Sullivan, se oponen terminantemente a ella. La Asociación Médica Norteamericana ha desatado una campaña publicitaria de desprestigio, afirmando que un sistema socializado no responde a los principios de libertad y oportunidad de la sociedad norteamericana (Washington Post, 27 de noviembre de 1990:H-12; 20 de febrero de 1991:A-1; 17 de marzo de 1991:23). Los mismos proponentes aceptan que podrían no ser considerada en el congreso cuando se discuta el asunto.

B) Otra propuesta traslada la obligación de los empleadores. La Legislación para un Seguro Nacional de Salud (National Health Insurance Legislation) forzaría a los empleadores ya sea a proporcionar cobertura 
médica a sus empleados y dependientes o a pagar un impuesto para un fondo de seguro gubernamental, agregando medidas que aseguren un control rígido de costos. Este plan está siendo elaborado por los comités de trabajo y finanzas del senado, apoyados en un plan elaborado en 1990 por la llamada Comisión Pepper (Washington Post, 20 de febrero 1991:A-4).

C) Por su parte, congresistas republicanos presentaron un plan de importantes reformas al mercado privado de seguros, bajo el cual 20 millones de empleados de empresas pequeñas y sus dependientes podrían obtener cobertura. La ley obligaría a negocios que emplean entre 3 y 25 trabajadores a hacer los arreglos necesarios para que éstos pudieran comprar, en grupo, un seguro básico de salud, incluyendo beneficios médicos y hospitalarios. El empleador podría pagar por el plan, si así lo deseara, ya que las primas se reducirían un $40 \%$ respecto de las actuales. Asimismo se impediría que las aseguradoras cancelen o se nieguen a renovar estas pólizas y se impondrían límites en posibles aumentos al precio de las mismas (Washington Post, 22 de marzo de 1991:A-23).

D) Por su parte, los propios estados están tomando medidas para enfrentar el problema. Las expansiones federales a MEDICAID, cuyos costos recayeron en los estados, obligaron a éstos a demandar dos años de congelamiento de tales expansiones. En ese tiempo, esperan generar un plan de acción que prevenga más gastos presupuestarios a su cargo. Mientras tanto, han planteado sus propios modelos de reforma. Algunos exploran versiones en miniatura de un sistema de cobertura universal, otros exploran la cobertura obligatoria por parte de los empleadores ya sea mínima o completa, dependiendo del tipo de empleado (Kosterlitz, 1990:708-713). Otros pretenden ajustar los programas MEDICAID y MEDICARE. Por ejemplo, mientras el estado de California ha sido duramente criticado por pugnar por un modelo de atención de cobertura universal, el estado de Oregon propuso una lista de enfermedades priorizada, el programa MEDICAID financiaría sólo los procedimientos mencionados, en los primeros lugares de la lista, a todos los solicitantes del estado. Dichos lugares dan preferencia a las enfermedades tratables que amenazan la vida así como a actividades de prevención. Condiciones no graves o aquellas fatales e incurables no tienen prioridad. Los críticos de este plan señalan que establece dos sistemas médicos, uno para aquéllos con acceso a los costosos milagros de la ciencia médica, y otro para quienes carecen de recursos para ello (New York Times, 22 de febrero de 1991:A-11).

E) Existen también propuestas particulares como la de la Fundación Heritage, ésta rechaza la idea de un seguro nacional de salud, o la de obligar a los patrones a pagar por el seguro de sus empleados. En su opinión, una reforma integral de las diferentes partes del sistema es lo apropiado, pero 
es responsabilidad de cada familia el protegerse obteniendo un seguro. Para ello, sería necesario reformar el código de impuestos a manera de beneficiar a los individuos por encima de las empresas y para motivar a los primeros a adquirir un seguro, independientemente de su tipo de trabajo. Lo anterior daría un incentivo a los individuos para pagar los costos menores de atención directamente y dejar el seguro sólo para gastos mayores. Esto a su vez incrementaría la competencia en la industria de la salud y mantendría los costos bajo control (Butler, 1989). Pero, la idea de que el libre juego de las fuerzas del mercado y la competencia por sí solos resolverán el problema, no es consecuente. La provisión de servicios médicos es el monopolio de un grupo y los consumidores de los servicios no están en posición de alegar sobre el costo (Beverly, 1987:Cap. 6).

Por su parte, Louise B. Russell propone un sistema unificado de atención a la salud para los pobres. Afirma que, ante la evidencia del fracaso de los programas gubernamentales en dar cobertura extensiva a toda la población de escasos recursos, es necesario un sistema nacional y comprensivo con los mismos servicios para todos aquellos ubicados por debajo de la línea de pobreza fijada oficialmente. La atención estaría subrogada a particulares o se prestaría en hospitales y clínicas del gobierno (Russell, 1989). Sus críticos afirman que es también una propuesta de servicios diferenciales, mientras que la parte gubernamental alude a la falta de recursos monetarios para sufragar la unificación del sistema.

Como se observa, existe una infinidad de propuestas de solución, sin embargo, ninguna cuenta con el apoyo decisivo necesario para su implementación. Es patente la importancia política que el tema ha adquirido en los años recientes, pero es poca la esperanza de ver el problema resuelto a corto plazo, tanto por los poderosos intereses afectados como por la falta de una cultura política de la salud que motive a la sociedad norteamericana a combatirlos seriamente.

\section{CONCLUSIONES}

El sistema de organización de los servicios de salud en los Estados Unidos revela una paradoja: siendo un país que cuenta con conocimientos, infraestructura y disponibilidad de recursos ilimitados para dar atención a todos sus ciudadanos, limita el acceso de una parte de éstos a sus servicios y cuando les proporciona el servicio, éste es diferencial en cuanto a calidad y extensión debido a que está condicionado por la capacidad de pago.

La mezcla confusa de programas privados y gubernamentales de atención a la salud abre una brecha que afecta a un número cada vez mayor de ciudadanos norteamericanos. La problemática de los costos es la punta 
del iceberg que revela la incapacidad del sistema para dar prioridad a la necesidad colectiva por encima de los intereses privados. La pregunta que se suprime es ¿ en quién recae la responsabilidad de proporcionar el nivel adecuado de atención para todos? Ello demuestra la falta de una cultura política de la salud en los Estados Unidos. Esto es, no existen mecanismos de participación efectiva de la comunidad para definir la orientación de sus servicios de salud (López, 1982:95-140).

La opinión pública norteamericana está a favor de la atención básica a la salud como un derecho de todos los ciudadanos, pero es renuente a aceptar una carga impositiva mayor para sufragar el costo de dicho sistema. El gobierno se muestra incapaz de asegurar ese derecho y se opone a su legalización. Los proveedores privados afirman que el problema no es suyo y, sin embargo, se les ha hecho pagar por él. Todos aceptan la necesidad de reformas pero nadie está dispuesto a pagar los costos que cualquier reforma implica. Mientras tanto, los mayormente afectados serán los pobres, ya que la mayor parte de las propuestas de control de costos tienen como consecuencia reducir o negar los servicios a esa población.

En el fondo, queda la evidencia de que la atención a la salud en los Estados Unidos es un monopolio ejercido por los proveedores privados con la anuencia del gobierno; este monopolio se rehúsa a disminuir sus ganancias en beneficio de la colectividad y se opone, y se opondrá, con toda la fuerza de sus organizaciones a cualquier intento que afecte sus intereses.

Así, la crisis que afecta al sistema de salud en los Estados Unidos no puede ser calificada como temporal. Es un problema estructural sin solución por la vía de reformas a ciertas leyes, reformando los programas vigentes o con el establecimiento de nuevos programas. Tampoco es un problema reciente, ya en 1971 se hablaba de una crisis en el sector salud y se llegaba a la misma conclusión de los proponentes de un sistema nacional de salud, pero que no es cabalmente aceptada en la sociedad norteamericana: sólo una política global y un programa nacional podrían provocar los cambios necesarios (Terris, 1980:109-117).

El aumento en los costos de la atención es una de las causas más importantes para propugnar por un programa nacional. Ese aumento es debido a la demanda de servicios para un número limitado de proveedores en un mercado que no admite ninguna restricción. A su vez, la costosa infraestructura hospitalaria no es racionalmente utilizada. Duplicación de servicios, subutilización de servicios más complejos, y ante incentivos y presiones cconómicas, la sobreutilización de los mismos contribuyen ampliamente a agravar el problema. Un programa nacional de salud implicaría racionalizar la prestación de servicios y su distribución desigual, 
pero tendería a reducir las ganancias que se obtienen del actual sistema. Y este es el obstáculo principal y casi insalvable que ocupará el debate sobre la salud en los noventa.

\section{BIBLIOGRAFÍA}

BEVERLY, David \& Edward Mc Sweeney. 1987. Social Welfare and Social Justice. Prentice Hall. USA.

BUTLER, Stuart M. 1989. Assuring Affordable Health Care For All Americans. The Heritage Foundation. Washington, D.C., no. 218. COTTINGHAM, Phoebe H. \& David T. Ellwood. (Eds.). 1990. Welfare Policy For The 1990's. Massachusetts. Harvard University Press.

KIRK, Víctor. 1990. "Gut Issue”, National Journal. Washington D.C., marzo 29, no.12.

KOSTERLITZ, Julie. 1990. "Seeking The Cure", National Journal. Washington D.C., marzo 29, no.12.

LÓPEZ Acữa, Daniel. 1982. La Salud Desigual en México. México, s. XXI. PIVEN, Frances \& Richard A. Cloward. 1982. The New Class War. New York. Pantheon Books.

RUSSELL, Louise. 1989. "Proposed: A Comprehensive Health Care System For The Poor". The Brookings Review. Washington D.C.

TALAVERA, Rosa. s/f. "Ocho años de reaganomía" Cuadernos semestrales. 23-24. CIDE. México.

TERRIS, Milton. 1980. La revolución epidemiologica y la medicina social. México, s. XXI.

\section{DOCUMENTOS:}

MAJOR LEGISLATION OF THE CONGRESS. CRS. Summary Issue/ 101st Congress. December 1990. MLC-64/MLC-67.

\section{PERIODICOS Y REVISTAS:}

New York Times:

3 de septiembre de 1990: A-1

4 de noviembre de 1990: 14

29 de noviembre de 1990: A-1

24 de diciembre de 1990: A-1

22 de febrero de 1991: A-11

24 de febrero de 1991: F-12

23 de marzo de 1991: A-1 


\section{Wall Street Juornal}

15 de mayo de 1990: B-1

\section{Washington Post:}

26 de febrero de 1991. "Lobbyits Roster Swells for Health Care Debate".

22 de noviembre de 1990:A-10. "Health Insurance Grapple With 'Fairness'".

27 de noviembre de 1990: H-7; H-12

30 de noviembre de 1990: A-3

15 de enero de 1991, Health Suplement: 7

20 de febrero de 1991: A-1; A-4

28 de febrero de 1991: A-18 (Editorial)

17 de marzo de 1991: A-23

22 de marzo de 1991: A-23

27 de marzo de 1991: A-21

2 de abril de 1991: A-19

8 de abril de 1991: D-1 